\title{
Characterization of MK6240, a tau PET tracer, in autopsy brain tissue from Alzheimer's disease cases
}

\author{
Mona-Lisa Malarte $^{1} \cdot$ Agneta Nordberg $^{1,2} \cdot$ Laetitia Lemoine $^{1}$
}

Received: 6 June 2020 / Accepted: 31 July 2020 / Published online: 24 September 2020

(C) The Author(s) 2020

\begin{abstract}
Purpose MK6240 is a second-generation tau PET tracer designed to detect the neurofibrillary tangles in the brains of patients with Alzheimer's disease (AD). The aim of the study was to characterize ${ }^{3} \mathrm{H}-\mathrm{MK} 6240$ in $\mathrm{AD}$ and control brain tissue and to compare its binding properties with those of first-generation tau PET tracers.

Methods Saturation binding assays with ${ }^{3} \mathrm{H}-\mathrm{MK} 6240$ were carried out in the temporal and parietal cortices of AD brains to determine the maximum number of binding sites (Bmax) and the dissociation constants $(\mathrm{Kd})$ at these sites. Competitive binding assays were carried out between ${ }^{3} \mathrm{H}-\mathrm{MK} 6240$ and unlabelled MK6240, AV-1451 (aka T807, flortaucipir) and THK5117, and between ${ }^{3} \mathrm{H}$-THK5351 and unlabelled MK6240. Regional binding studies with ${ }^{3} \mathrm{H}-\mathrm{MK} 6240$ were carried out in homogenates from six $\mathrm{AD}$ and seven control brains and, using autoradiography, on large frozen sections from two $\mathrm{AD}$ brains and one control brain.

Results The saturation binding assays gave Bmax and $\mathrm{Kd}$ values of $59.2 \mathrm{fmol} / \mathrm{mg}$ and $0.32 \mathrm{nM}$ in the temporal cortex and $154.7 \mathrm{fmol} / \mathrm{mg}$ and $0.15 \mathrm{nM}$ in the parietal cortex. The competitive binding assays revealed two binding sites with affinities in the picomolar and nanomolar range shared by ${ }^{3} \mathrm{H}-\mathrm{MK} 6240$ and all the tested unlabelled compounds. There were no binding sites in common between ${ }^{3} \mathrm{H}$-THK5351 and unlabelled MK6240. Regional binding of ${ }^{3} \mathrm{H}-\mathrm{MK} 6240$ was significantly higher in $\mathrm{AD}$ brain tissue than in controls. Binding in brain tissue from AD patients with early-onset AD was significantly higher than in brain tissue from patients with late-onset AD. Binding of ${ }^{3} \mathrm{H}-\mathrm{MK} 6240$ was not observed in off-target regions. Autoradiography showed high regional cortical binding in the two AD brains and very low binding in the control brain.

Conclusions ${ }^{3} \mathrm{H}-\mathrm{MK} 6240$ has a high binding affinity for tau deposits in AD brain tissue but also has different binding characteristics from those of the first-generation tau tracers. This confirms the complexity of tau tracer binding on tau deposits with different binding affinities for different binding sites.
\end{abstract}

Keywords Alzheimer's disease · Tau imaging · Autoradiography $\cdot$ MK6240 · AV-1451 · THK5117

This article is part of the Topical Collection on Neurology.

Electronic supplementary material The online version of this article (https://doi.org/10.1007/s00259-020-05035-y) contains supplementary material, which is available to authorized users.

Laetitia Lemoine

laetitia.lemoine@ki.se

1 Department of Neurobiology Care Sciences and Society, Division of Clinical Geriatrics, Center of Alzheimer Research, Karolinska Institutet, Stockholm, Sweden

2 Theme Aging, Karolinska University Hospital, Stockholm, Sweden

\section{Introduction}

Alzheimer's disease (AD) is characterized by the extracellular amyloid beta accumulation in the form of amyloid plaques and abnormal intracellular accumulation of tau protein into neurofibrillary tangles (NFTs) in the brain, both of which lead to progressive neurodegeneration [1]. The abnormal accumulation of tau protein is a characteristic of several other diseases which can be grouped under the generic name tauopathies [2]. The range of clinical phenotypes of these tauopathies can make clinical diagnosis very challenging [3], and there is a need for new tools to help understand and differentiate between them in vivo in the early stages of the disease. 
PET tracers for studying the progression of tau deposits in vivo have been synthesized. However, this has been challenging because the deposits are also intracellular: the tau PET tracers need to be able to cross both the blood-brain barrier and the cell membrane. Moreover, the method of tau inclusion varies with the tauopathy; exon 10 may be included or excluded during the alternative splicing of the tau gene, leading to three-repeat (3R) or four-repeat (4R) tau isoforms [4]. The first generation of tau PET tracers, including THK family compounds and AV-1451 (also known as T807, flortaucipir), has been widely explored in vivo in AD patients, and its effects have been compared with those in controls; this has generated important information about the progression of tau pathology and its relation to functional changes in patients (see reviews [5-7]). In vitro characterization of the first-generation tracers has also been extensively carried out. In vitro binding assays have indicated that ${ }^{3} \mathrm{H}$-THK5117 has good binding properties, with high affinity and specificity for tau in post-mortem brain tissue from AD patients [8]. Using autoradiography techniques and AT8 immunostaining on adjacent paraffin sections from an AD brain, we have previously observed similar regional distributions of ${ }^{3} \mathrm{H}$-THK5117 and tau [8]. Binding assays and competitive studies of first-generation tau PET tracers indicated that THK5117, THK5351 and AV-1451 targeted the same binding sites with different affinities, whereas PBB3 seemed to have its own target [9, 10]. In 2017, Fitzpatrick et al. used cryo-electron microscopy to describe the structure of tau fibrils. In silico studies using this structure have suggested the presence of multiple theoretical binding sites for which the first generation of tau tracers had differing affinities [11]. Unfortunately, first-generation tau PET tracers also demonstrated off-target binding: THK5117, THK5351 and AV-1451 bind to monoamine oxidase B (MAO-B) [12], AV-1451 binds to neuromelanin [13] and PBB3 binds to amyloid plaques and alpha-synuclein $[14,15]$. To avoid this scenario, a second generation of tau PET tracers has been designed; these include RO948, PI2620, JNJ311, MK6240, PM-PBB3 and AM-PBB3 [16]. The results of preliminary studies are promising for many of these. RO948 has good in vivo kinetic distribution and brain uptake, with low off-target binding [17]; PI2620 has a high affinity for pathological tau aggregates and has entered clinical trials [18]; results for JNJ311 are promising in trials of rodent and non-human primate tissue [19]; MK6240 binds specifically to one site on NFT-rich AD brain tissue and neither binds to off-target sites nor has an affinity for amyloid plaques [20-22]. In silico data have suggested that MK6240, JNJ311 and PI2620 have a low affinity for MAO-B [23].

An in vitro study of the second-generation MK6240 tracer has shown a high binding affinity for brain homogenate rich in NFTs [21, 24]. Similarly, a recent in vivo pilot study by Lohith et al. in AD patients showed that MK6240 binding occurred in regions rich in NFT deposits [25]; More recently, Pascoal et al. reported that ${ }^{18}$ F-MK6240 could be used to differentiate early and late disease stages of $\mathrm{AD}$ as well as discriminating AD from cognitively unimpaired and frontotemporal dementia [26]. In silico modelling has suggested the possibility of four theoretical binding sites on tau fibrils for MK6240 [27].

In this study, our aim was to characterize the ${ }^{3} \mathrm{H}-\mathrm{MK} 6240$ binding pattern in human autopsy brain tissue from AD cases and to compare this with binding in brain tissue from age-matched controls. We used binding assays in brain homogenates and autoradiography on large frozen hemispherical sections and compared the results with the binding properties of several first-generation tau PET tracers.

\section{Materials and methods}

\section{Chemicals}

${ }^{3} \mathrm{H}-\mathrm{MK} 6240$ (specific activity $(\mathrm{SA})=45.77 \mathrm{Ci} / \mathrm{mmol}$ ) and unlabelled MK6240 were provided by Merck Sharp and Dohme Corp. (Whitehouse Station, USA). ${ }^{3}$ H-THK5351 $(\mathrm{SA}=35 \mathrm{Ci} / \mathrm{mmol})$ was synthesized and labelled at the Centre for Psychiatric Research, Department of Clinical Neuroscience, Karolinska Institutet (Solna, Sweden). Unlabelled AV-1451 was synthesized by He Tian (Institute of Fine Chemicals, East China University of Science and Technology, Shanghai, China). Unlabelled THK5117 was custom synthesized by Novandi (Södertalje, Sweden).

\section{Brain tissue}

Frozen post-mortem tissue from eight $\mathrm{AD}$ and seven control brains from the Netherlands Brain Bank (NBB) was homogenized in phosphate buffer saline (PBS) with additional $0.1 \%$ bovine serum albumin (BSA; $250 \mathrm{mg} / \mathrm{mL}$ ) and protease/ phosphatase inhibitors $(10 \mu \mathrm{L} / \mathrm{mL})$.

Large hemispherical frozen post-mortem brain sections (100 $\mu \mathrm{m}$ thick) were obtained from two patients with $\mathrm{AD}$ who had been clinically followed by one of the authors (A. N.) at the Department of Geriatric Medicine, Karolinska University Hospital Huddinge, Sweden, and one control.

Table 1 provides a summary of demographic information about the patients and controls from which brain tissue was sourced.

\section{Binding assays}

Optimal binding assay conditions were established for ${ }^{3} \mathrm{H}$-MK 6240 by varying the quantity of the brain homogenate $(0.2,0.5,0.75,1$ or $2 \mathrm{mg} / \mathrm{mL})$ and the incubation temperature $\left(25^{\circ} \mathrm{C}, 37^{\circ} \mathrm{C}\right.$ ) (see Supplemental Fig. 1). 
Table 1 Demographic information about the patients and controls from whom brain tissue was obtained for binding assays (AD case numbers 18) and autoradiography studies (AD patients $\mathrm{A}$ and $\mathrm{B}$ and control $\mathrm{C}$ ). The sex, age at death, apolipoprotein (ApoE) genotype, Braak stage at cerebral pathological assessment, presence of early- or late-onset $\mathrm{AD}$ and post-mortem delay are provided

\begin{tabular}{|c|c|c|c|c|c|c|c|}
\hline & Case no. & $\operatorname{Sex}(\mathrm{M} / \mathrm{F})$ & Age (years) & ApoE (E/E) & Braak stage & EOAD/LOAD & Post-mortem delay (hours:minutes) \\
\hline \multirow[t]{11}{*}{ AD patients } & 1 & $F$ & 59 & $4 / 4$ & 5 & $E O A D$ & $04: 20$ \\
\hline & 2 & $\mathrm{~F}$ & 66 & $4 / 3$ & 5 & EOAD & $06: 30$ \\
\hline & 3 & M & 70 & $4 / 4$ & 4 & EOAD & 04:00 \\
\hline & $* 4$ & $M$ & 77 & $N A$ & 6 & $L O A D$ & $06: 35$ \\
\hline & 5 & $M$ & 78 & $4 / 4$ & 5 & $L O A D$ & $06: 35$ \\
\hline & 6 & $\mathrm{~F}$ & 81 & $4 / 3$ & 5 & LOAD & $06: 15$ \\
\hline & $* 7$ & $\mathrm{~F}$ & 82 & NA & 6 & LOAD & 04:20 \\
\hline & $* 8$ & $\mathrm{~F}$ & 85 & $3 / 3$ & 4 & LOAD & 06:00 \\
\hline & Mean & $3 \mathrm{M} / 5 \mathrm{~F}$ & $72 \pm 13$ & $\begin{array}{l}5 \mathrm{E} 4 \\
1 \mathrm{E} 3\end{array}$ & $4-6$ & $3 \mathrm{EOAD} / 5 \mathrm{LOAD}$ & \\
\hline & A & $\mathrm{F}$ & 79 & $4 / 4$ & 5 & LOAD & $16: 00$ \\
\hline & $\mathrm{B}$ & M & 81 & $4 / 4$ & 6 & LOAD & $17: 00$ \\
\hline \multirow[t]{9}{*}{ Controls } & 9 & $\mathrm{~F}$ & 50 & $3 / 3$ & 1 & N/A & $04: 10$ \\
\hline & 10 & M & 62 & $3 / 3$ & 1 & N/A & $07: 20$ \\
\hline & 11 & $\mathrm{~F}$ & 71 & $3 / 3$ & 1 & N/A & $07: 10$ \\
\hline & 12 & $\mathrm{~F}$ & 77 & $3 / 3$ & 1 & N/A & $02: 55$ \\
\hline & 13 & M & 78 & $3 / 3$ & 1 & N/A & $17: 40$ \\
\hline & 14 & M & 79 & $3 / 3$ & 2 & N/A & 09:00 \\
\hline & 15 & $\mathrm{~F}$ & 84 & $3 / 3$ & 1 & N/A & $06: 55$ \\
\hline & Mean & $3 \mathrm{M} / 4 \mathrm{~F}$ & $67 \pm 17$ & $\begin{array}{l}7 \mathrm{E} 3 \\
0 \mathrm{E} 4\end{array}$ & $1-2$ & & \\
\hline & $\mathrm{C}$ & $\mathrm{F}$ & 76 & & 1 & $N / A$ & 04:00 \\
\hline
\end{tabular}

Values in italics indicate AD patients (numbers 1, 4, 5) whose temporal lobes were used for the saturation and competition studies, and the 59-year-old $\mathrm{AD}$ patient (number 1) whose parietal lobe was used. The asterisks ' $*$ ' indicate AD patients (numbers $4,7,8$ ) whose hippocampus was used for the competition studies

$A D$, Alzheimer's disease; $E O A D$, early-onset $\mathrm{AD} ; F$, female; $L O A D$, late-onset $\mathrm{AD} ; M$, male; $N / A$, not applicable

Saturation binding assays were carried out using homogenates of the temporal and parietal cortices from AD cases $(0.2 \mathrm{mg} / \mathrm{mL})$, with increasing concentrations of ${ }^{3} \mathrm{H}-\mathrm{MK} 6240$ (0.05-2 $\mathrm{nM}$ ) incubated for $90 \mathrm{~min}$ at $37^{\circ} \mathrm{C}$ in $\mathrm{PBS}+0.1 \%$ BSA (pH 7.4). The extent of non-specific binding was determined using $1 \mu \mathrm{M}$ unlabelled MK6240. The binding reaction was terminated by filtering the sample through glass fibre filters (which had been pre-soaked for at least $3 \mathrm{~h}$ in $0.3 \%$ polyethylenimine solution) and washing it three times with cold binding assay buffer. The extent of ${ }^{3} \mathrm{H}-\mathrm{MK} 6240$ binding was quantified using a scintillation counter (Beckman Coulter LS6500). The equilibrium dissociation constant (Kd) and the maximum number of binding sites (Bmax) were determined using GraphPad Prism software version 8 for Mac OSX.

Competitive binding assays with ${ }^{3} \mathrm{H}-\mathrm{MK} 6240$ (0.5 nM) were carried out for $90 \mathrm{~min}$ at $37{ }^{\circ} \mathrm{C}$ on temporal cortex homogenates from two AD brains, in PBS $+0.1 \%$ BSA (pH 7.4), using increasing concentrations of unlabelled MK6240, unlabelled THK5117 or unlabelled AV-1451 $\left(1.10^{-14}-1.10^{-5} \mathrm{M}\right)$. The binding assay was terminated as described above. The half-maximal effective concentration (IC50) was determined using GraphPad Prism software and data were analysed using a 2-site competition model.

Competitive binding assays were also carried out with ${ }^{3} \mathrm{H}$-THK5351 (1.5 nM) in PBS + 0.1\% BSA ( $\mathrm{pH} 7.4$ ) on hippocampus homogenates, using increasing concentrations of unlabelled MK6240 (1.10 $\left.0^{-14}-1.10^{-5} \mathrm{M}\right)$ in PBS $+0.1 \%$ BSA for $2 \mathrm{~h}$ at room temperature.

The regional distribution of ${ }^{3} \mathrm{H}-\mathrm{MK} 6240(0.5 \mathrm{nM})$ to seven brain regions (frontal, temporal and parietal cortices, hippocampus, thalamus, caudate nucleus and cerebellum) was investigated using tissue from six AD patients (three with early-onset AD (EOAD, <65 years old) and three with late-onset $\mathrm{AD}$ (LOAD, > 65 years old)) and seven age-matched controls. The extent of non-specific binding was determined using unlabelled MK6240 (1 $\mu \mathrm{M})$. Data were analysed using GraphPad Prism software. ${ }^{3} \mathrm{H}-\mathrm{MK} 6240$ binding in $\mathrm{AD}$ brain tissue was compared with that in control brain tissue using a 2-way ANOVA test with Graphpad Prism 8 software. 


\section{Autoradiography binding studies in Alzheimer's disease and control autopsy brain tissue}

A regional distribution study of ${ }^{3} \mathrm{H}-\mathrm{MK} 6240$ using autoradiography on large frozen brain sections was also carried out. After being allowed to dry, the sections were pre-incubated for $15 \mathrm{~min}$ in PBS + 0.1\% BSA ( $\mathrm{pH} 7.4$ ) before incubation for $1 \mathrm{~h}$ with ${ }^{3} \mathrm{H}-\mathrm{MK} 6240(0.5-1 \mathrm{nM})$ at room temperature. The extent of non-specific binding was determined using unlabelled MK6240 $(1 \mu \mathrm{M})$. After $1 \mathrm{~h}$, the sections were washed three times for $5 \mathrm{~min}$ in cold binding assay buffer $\left(4^{\circ} \mathrm{C}\right)$ and briefly dipped into cold de-ionized water $\left(4^{\circ} \mathrm{C}\right)$. After overnight drying, they were put into a phospho-image cassette with a phospho-plate apposed onto the sections for 7 days, with a tritiated standard. The plates were then scanned with the BAS-2500 phosphor imager and regions of interest were drawn manually using multigauge software.

\section{Results}

Saturation binding assay results for the AD brain tissue are shown in Fig. 1. The mean saturation binding curve in the temporal cortices of two AD cases is shown in Fig. 1a. Using a ${ }^{3} \mathrm{H}-\mathrm{MK} 6420$ concentration range from 0.05 to $2 \mathrm{nM}$, we observed a Bmax of $59.2 \mathrm{fmol} / \mathrm{mg}$ and a $\mathrm{Kd}$ of $0.32 \mathrm{nM}$. In the same experiment carried out in the parietal cortex of one AD brain (case 1), we observed a Bmax of $154.7 \mathrm{fmol} / \mathrm{mg}$ and a $\mathrm{Kd}$ of $0.15 \mathrm{nM}$. Data transformed in a Scatchard plot suggested one binding site in both regions.

The competitive binding assay between ${ }^{3} \mathrm{H}-\mathrm{MK} 6240$ and unlabelled MK6240 (Fig. 2a) showed two relevant binding sites: the unlabelled competitor had a super-high affinity for site 1 , in the picomolar range: $\mathrm{IC}_{50(\text { supHigh })}=1 \mathrm{pM}$ and a high affinity for site 2 , in the nanomolar range: $\mathrm{IC}_{50(\mathrm{High})}=12 \mathrm{nM}$. AV-1451 and THK5117 were also in competition with ${ }^{3} \mathrm{H}-\mathrm{MK} 6240$ at two binding sites: $\mathrm{IC}_{50(\text { supHigh })}=0.1 \mathrm{pM}$, $\mathrm{IC}_{50 \text { (High) }}=2 \mathrm{nM}$ and $\mathrm{IC}_{50 \text { (supHigh) }}=2 \mathrm{pM}, \mathrm{IC}_{50 \text { (low) }}=$ $304 \mathrm{nM}$, respectively. The proportion of binding sites for which the tracers had a super-high affinity (corresponding to IC50 (supHigh) is shown in Table 2. In the competitive studies between unlabelled MK6240 and ${ }^{3} \mathrm{H}-\mathrm{MK} 6240$, the unlabelled competitor had a super-high affinity for $58 \%$ of the sites and a high affinity for $42 \%$ of the sites. AV-1451 had a super-high affinity for the highest proportion of sites (74\%) when competing with ${ }^{3} \mathrm{H}-\mathrm{MK} 6240$, whereas the THK family compounds had the lowest values; THK5117 had a super-high affinity for $37 \%$ of the sites.

Figure $2 \mathrm{~b}$ shows the results of competitive binding studies using increasing concentrations of unlabelled MK6240 and ${ }^{3} \mathrm{H}$-THK5351. The unlabelled MK6240 did not compete with ${ }^{3} \mathrm{H}-\mathrm{THK} 5351$.

The regional distribution of ${ }^{3} \mathrm{H}-\mathrm{MK} 6240$ in $\mathrm{AD}$ and control brain homogenates is shown in Fig. 3. We detected low specific binding in the seven brain regions of all the control brains. ${ }^{3} \mathrm{H}-\mathrm{MK} 6240$ binding in the frontal, parietal and temporal cortices and hippocampus from AD brains was statistically significantly higher $(p<0.0001)$ than in these regions in control brains. No or low binding was observed in the thalamus, caudate nucleus and cerebellum in AD brains, with no significant differences from control brains (Fig. 3a). Figure $3 b$ shows the regional distribution when the data were analysed according to whether the patients had EOAD or LOAD. The binding of ${ }^{3} \mathrm{H}-\mathrm{MK} 6240$ was significantly higher in EOAD
Fig. 1 Saturation of binding sites in the temporal and parietal cortices of patients with Alzheimer's disease (AD). a Saturation curve for ${ }^{3} \mathrm{H}-\mathrm{MK} 6240$ $(0.05-2 \mathrm{nM})$ in temporal cortex brain homogenates from two $\mathrm{AD}$ patients (patients 4 and 5). The solid regression line was determined by Graphpad Prism software. Analyses using GraphPad Prism software showed one binding site. b Saturation curve for ${ }^{3} \mathrm{H}-\mathrm{MK} 6240(0.05$ $2 \mathrm{nM}$ ) in the parietal cortex brain homogenate from one $\mathrm{AD}$ patien (patient 1). Bmax = maximum number of binding sites; $\mathrm{Kd}=$ dissociation constant; $R^{2}=$ regression coefficient a

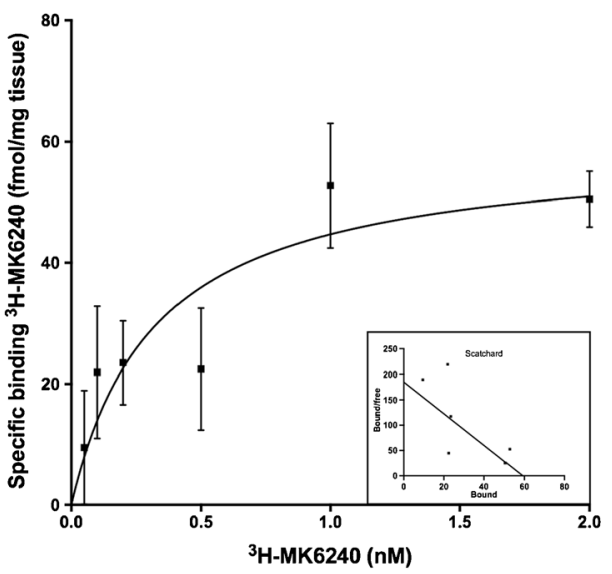

$B \max =59.2 \mathrm{fmol} / \mathrm{mg}$

$\mathrm{Kd}=0.32 \mathrm{nM}$

$R^{2}=0.45$ b

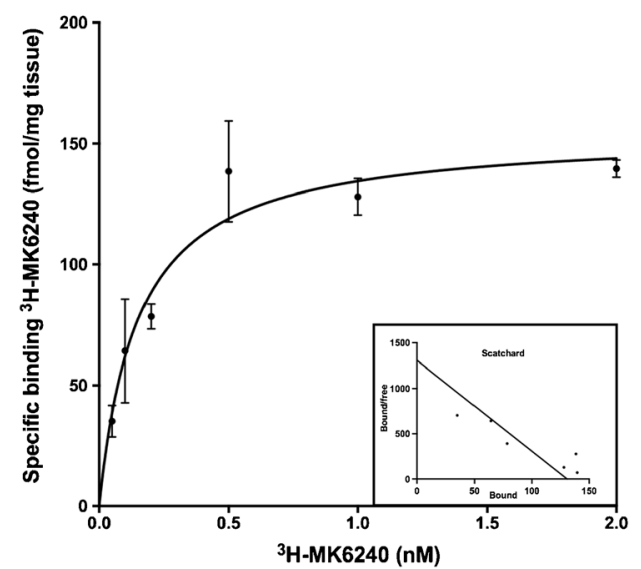

$B \max =154.7 \mathrm{fmol} / \mathrm{mg}$

$\mathrm{Kd}=0.15 \mathrm{nM}$

$R^{2}=0.85$ 
Table 2 Percentage of ${ }^{3} \mathrm{H}-$ MK6240 binding sites in the temporal cortex (or ${ }^{3} \mathrm{H}-\mathrm{THK} 5351$ binding sites in the hippocampus) for which the unlabelled compound (THK5117, AV-1451 or MK6240) had a super-high affinity in competitive studies

\begin{tabular}{lllr}
\hline Radioligand & Competitor & Fraction of sites with super-high affinity for the competitor $(\%)$ & $R^{2}$ \\
\hline${ }^{3}$ H-MK6240 & AV-1451 & 74 & 0.60 \\
& THK5117 & 37 & 0.60 \\
& MK6240 & 58 & 0.70 \\
${ }^{3}$ H-THK5351 & AV1451* & 35 & 0.96 \\
& THK5117* & 46 & 0.96 \\
& MK6240 & 0 & N/A \\
\hline
\end{tabular}

*See Lemoine et al. [12] for competitive studies using ${ }^{3}$ H-THK5351 versus unlabelled THK5117 or AV-1451 $N / A$, not applicable; $R^{2}$, regression coefficient brain tissue than in LOAD and control tissue in cortical regions (frontal, parietal and temporal cortices; $p<0.0001$ ) with the highest binding occurring in the temporal cortex. The binding was significantly higher in the EOAD hippocampus than in the control hippocampus $(p<0.0001)$. The binding was also significantly higher in LOAD tissue than in control tissue for the frontal cortex $(p=0.0084)$, temporal cortex $(p<0.0001)$ and hippocampus $(p<0.0001)$. There were no differences between EOAD and LOAD binding in the subcortical regions (thalamus and caudate nucleus).
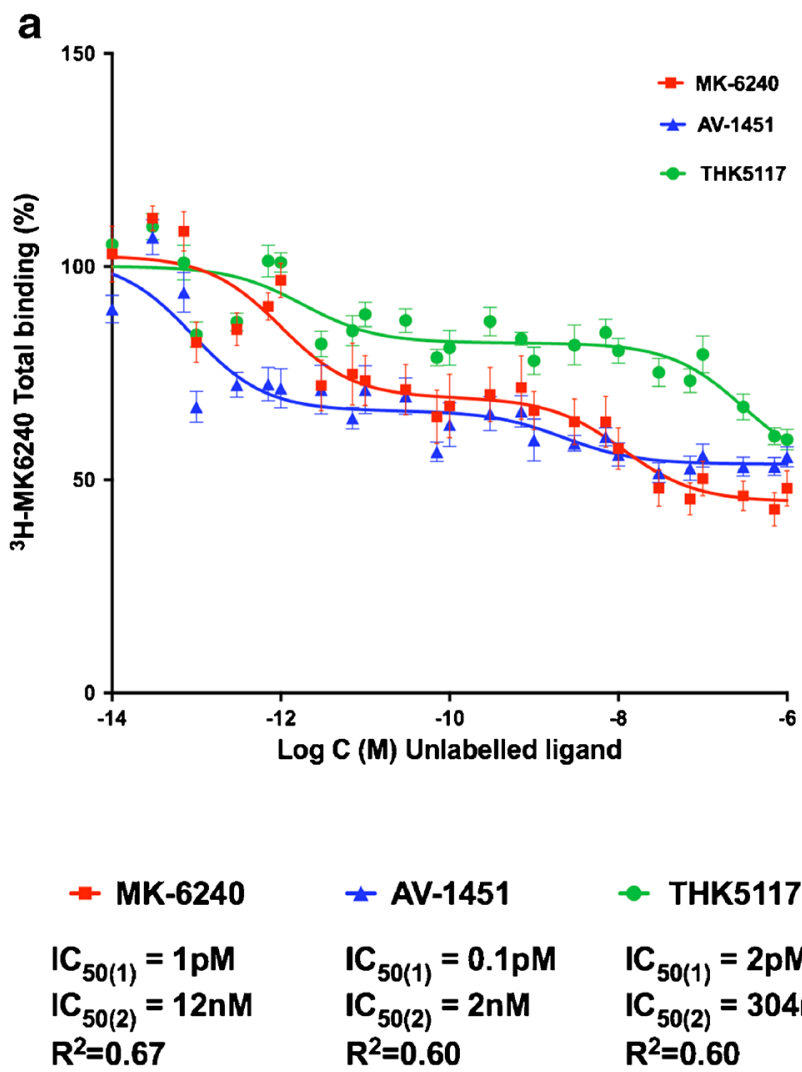

THK5117

$I C_{50(1)}=2 \mathrm{pM}$

$\mathrm{IC}_{50(2)}=304 \mathrm{nM}$ $\mathbf{R}^{\mathbf{2}}=\mathbf{0 . 6 0}$

Fig. 2 a Competitive binding assays between ${ }^{3} \mathrm{H}-\mathrm{MK} 6240(0.5 \mathrm{nM})$ and increasing concentrations of unlabelled MK6240, AV-1451 and THK5117 $\left(1.10^{-14}-1.10^{-6} \mathrm{M}\right)$. b Competitive binding assay in hippocampus tissue from the brains of three patients with Alzheimer's disease using ${ }^{3} \mathrm{H}$-THK5351 (1.5 nM) and increasing concentrations of MK6240

The autoradiography results for the regional distribution of ${ }^{3} \mathrm{H}-\mathrm{MK} 6240$ in AD patients A (79 years old) and B (81 years old) and control (76 years old) are presented in Fig. 4. The specific binding in femtomole per milligram is shown in Table 3. We observed binding in the frontal and temporal cortices with a clear laminar pattern in tissue from $\mathrm{AD}$ patient A (see enlargement in Fig. 4). The distribution in tissue from $\mathrm{AD}$ patient $\mathrm{B}$ was similar but the binding was less intense, as partly explained by the different pathology (as shown in AT8 staining; Fig. 4). There was high specific binding in the

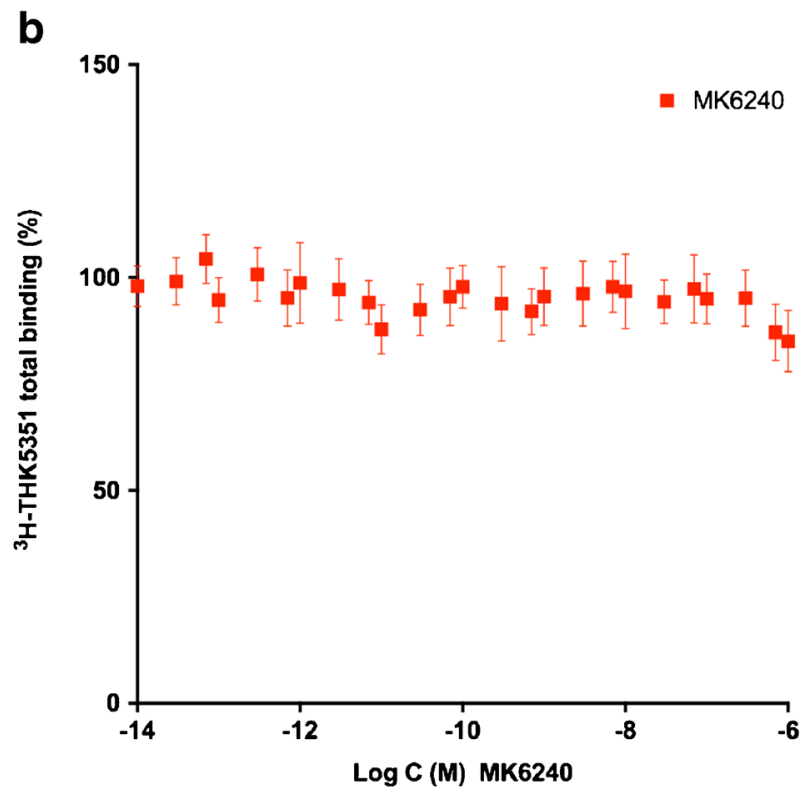

\section{Not converged}

$\left(1.10^{-14}-1.10^{-6} \mathrm{M}\right)$. Error bars represent the SEM from three experiments, each in triplicate, for each unlabelled compound. $\mathrm{C}=$ concentration; high = high-affinity binding site; IC50 = half-maximal effective concentration; low = low-affinity binding site; $R^{2}=$ regression coefficient; supHigh = super-high-affinity binding site 


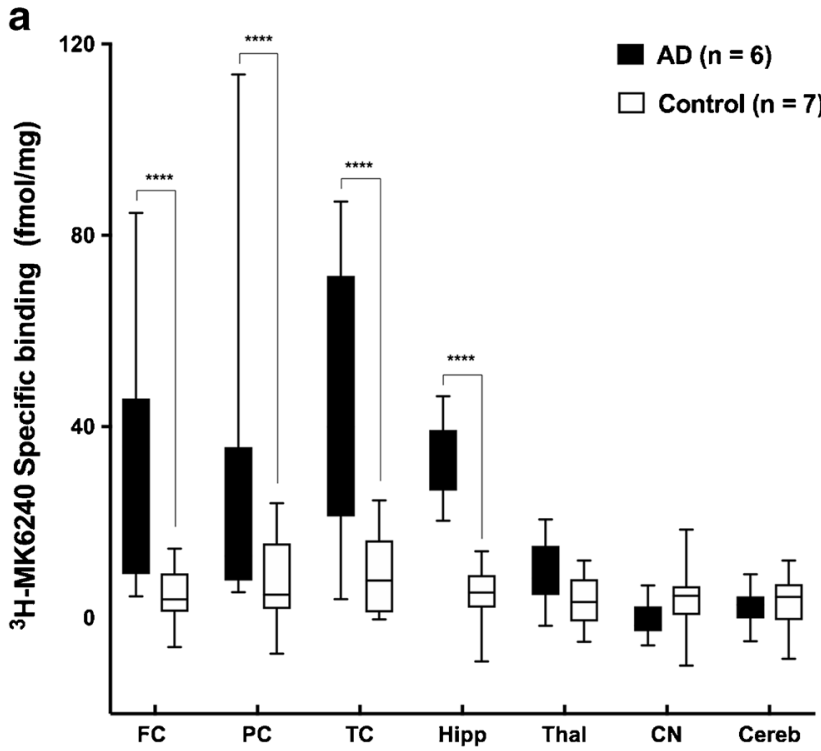

Fig. 3 Regional binding distribution of ${ }^{3} \mathrm{H}-\mathrm{MK} 6240$ in post-mortem brain homogenates from a six cases with Alzheimer's disease (AD) and seven controls and $\mathbf{b}$ the same six cases separated according to whether they had early-onset $\mathrm{AD}$ (EOAD; three cases) or late-onset AD (LOAD; three patients), with the same seven controls. The regions of interest were

entorhinal cortex from both brains $(90.2 \mathrm{fmol} / \mathrm{mg}$ for patient A and $128.4 \mathrm{fmol} / \mathrm{mg}$ for patient B). Similar high specific binding was found in the fusiform gyrus $(119 \mathrm{fmol} / \mathrm{mg}$ and $115.5 \mathrm{fmol} / \mathrm{mg}$ ). Case A showed lower binding in the temporal cortex $(49.6 \mathrm{fmol} / \mathrm{mg})$ than case B $(216.6 \mathrm{fmol} / \mathrm{mg})$. The binding was lower for both brains in the insular cortex and was lower in the hippocampus from patient A $(42.4 \mathrm{fmol} / \mathrm{mg})$ than in that from patient $B(81 \mathrm{fmol} / \mathrm{mg})$. The inverse was seen for the frontal cortex $(109.2 \mathrm{fmol} / \mathrm{mg}$ for patient $\mathrm{A}$ and $45.6 \mathrm{fmol} / \mathrm{mg}$ for patient B). Almost no binding was present in both total and NSP sections for the control.

\section{Discussion}

The aim of this study was to characterize and compare the binding properties of ${ }^{3} \mathrm{H}-\mathrm{MK} 6240$ in human post-mortem

Table 3 Specific binding of ${ }^{3} \mathrm{H}-\mathrm{MK} 6240$ (fmol/mg tissue) in regions of interest in two Alzheimer's disease (AD) brain sections (100 $\mu \mathrm{M}$ thick)

\begin{tabular}{lll}
\hline Regions of interest & AD patient A & AD patient B \\
\hline Hippocampus & 42.4 & 81.0 \\
Entorhinal cortex & 90.2 & 128.4 \\
Fusiform gyrus & 119.0 & 115.5 \\
Temporal cortex & 49.6 & 216.6 \\
Insula & 24.0 & 32.8 \\
Frontal cortex & 109.2 & 45.6 \\
Cingulate gyrus & 99.9 & 64.9 \\
\hline
\end{tabular}

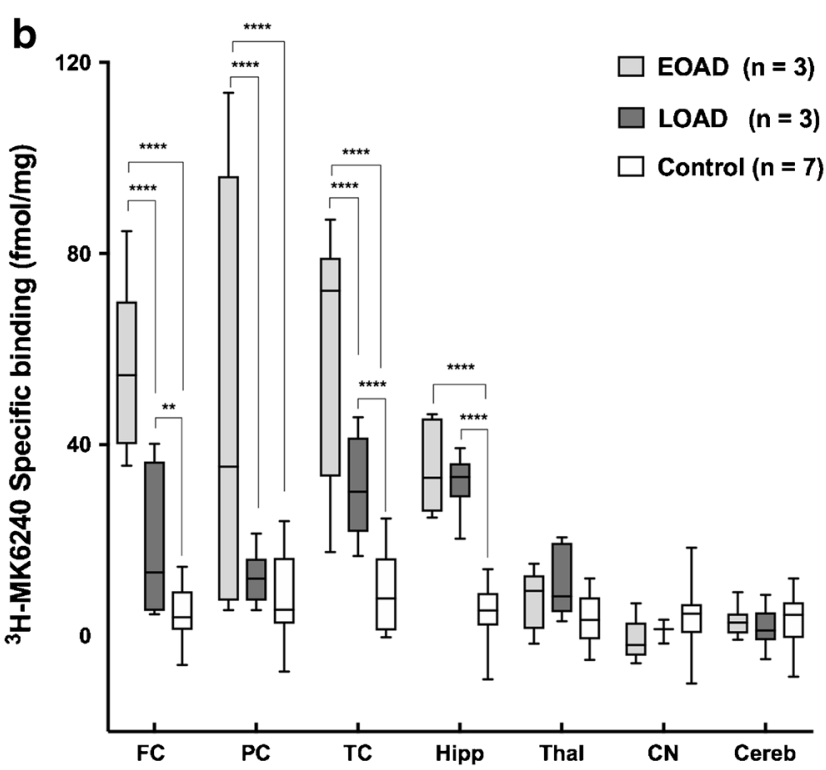

the frontal cortex (FC), parietal cortex (PC), temporal cortex (TC), hippocampus (Hipp), thalamus (Thal), caudate nucleus $(\mathrm{CN})$ and cerebellum (Cereb). Error bars represent the SEM from three experiments, each in triplicate, for each area. Prism software was used for a 2-way analysis of variance multiple comparisons. ${ }^{* * *} p<0.001, * * * * p<0.0001$

brain tissue from $\mathrm{AD}$ cases with its properties in nondemented control tissue and with the properties of the first-generation tau tracers in a head-to-head comparison study.

In saturation binding assays, we observed that ${ }^{3} \mathrm{H}-\mathrm{MK} 6240$ had a high binding affinity for tissue in the temporal cortices of two AD cases: Kd was $0.32 \mathrm{nM}$ and Bmax was $59.2 \mathrm{fmol} / \mathrm{mg}$. Interestingly, when we carried out the same experiment in the parietal cortex of an EOAD brain, we observed a Kd of $0.15 \mathrm{nM}$ and a Bmax of $154.7 \mathrm{fmol} / \mathrm{mg}$. The difference in Bmax between the temporal cortex and the parietal cortex might have been the result of different brains being used in the experiment; disease progression was severe and fast for the EOAD patient.

The Bmax of ${ }^{3} \mathrm{H}-\mathrm{MK} 6240$ was similar to that published by Hostetler et al. in 2016 [21], where Bmax ranged from $7.8 \mathrm{nM}$ to $93.4 \mathrm{nM}$ for ${ }^{3} \mathrm{H}-\mathrm{MK} 6240$ and $15 \mathrm{nM}$ to $119.7 \mathrm{nM}$ for ${ }^{3} \mathrm{H}-\mathrm{AV}-1451$. It has already been noted here that Bmax varies between brain regions. In general, the Kd of MK6240 indicates that the tracer has a higher affinity for $\mathrm{AD}$ tissue than the first-generation tracers THK5117 (2.2 nM) and AV-1451 (0.6-3.7) [7, 21]. THK5117 Bmax, earlier reported, seems to be higher $\left(\mathrm{Bmax}_{1}=250 \mathrm{fmol} / \mathrm{mg}, \mathrm{Bmax}_{2}=1416 \mathrm{fmol} / \mathrm{mg}[8]\right)$ compared with MK6240 Bmax values, which probably might reflect different binding site properties and/or non-specific targets. These findings are interesting and should be taken into consideration during the use of differing brain homogenates.

In order to obtain deeper insight into the binding properties of MK6240, we carried out competitive binding assays using a wide range of unlabelled compounds. Although the saturation binding assay showed only one binding site, competitive binding studies between unlabelled MK6240 and ${ }^{3} \mathrm{H}-\mathrm{MK} 6240$ in 


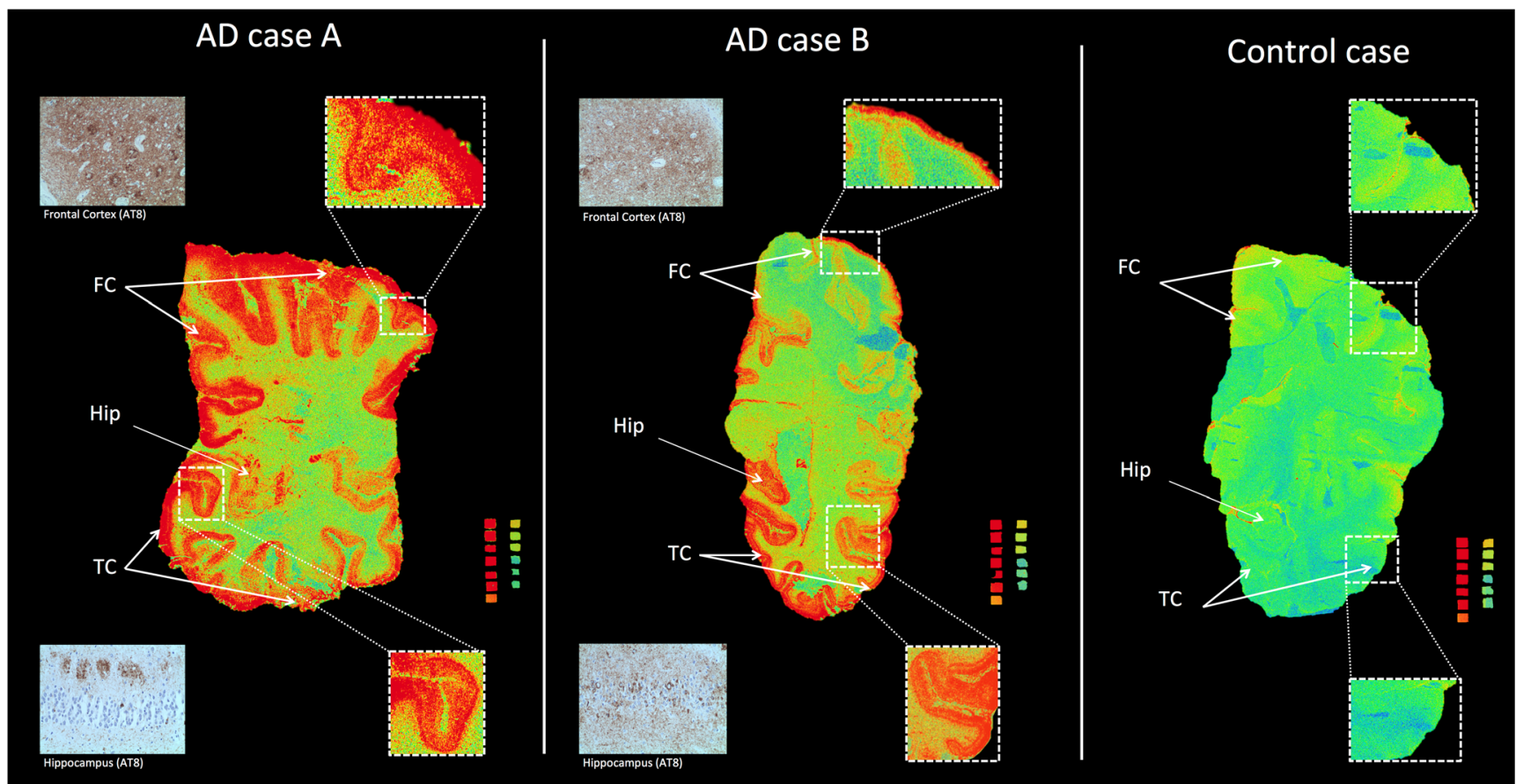

Fig. 4 Autoradiography of ${ }^{3} \mathrm{H}-\mathrm{MK} 6240$ (1 nM) binding on frozen adjacent sections from the post-mortem left brain hemispheres of Alzheimer's disease (AD) patients $\mathrm{A}$ and $\mathrm{B}$ and a control. The enlargements depict the cortical laminar patterns in the frontal cortex (FC) and temporal cortex

two AD brains revealed at least two binding sites for which the tracer had different binding affinities. We saw evidence of a super-high-affinity site in the picomolar range, a high-affinity site in the nanomolar range and a low-affinity site in the higher nanomolar range. There were two binding sites for which MK6240 and AV-1451 showed affinity (one super-high and one high affinity for each tracer) and two sites for which THK5117 showed affinity (one super-high and one low affinity). The proportional analysis of these sites revealed that MK6240 had a super-high affinity for 58\% of the sites. Competitive binding studies showed that both THK5117 and AV-1451 competed with ${ }^{3} \mathrm{H}-\mathrm{MK} 6240$ in a 2-site model and that a site in the picomolar range seems to be common to all the tracers. Unlabelled THK5117 competed with ${ }^{3} \mathrm{H}-\mathrm{MK} 6240$ at two sites, one with super-high affinity and one with low affinity. Unlabelled AV-1451 competed with ${ }^{3} \mathrm{H}-\mathrm{MK} 6240$ at two sites with very high affinity, one in the nanomolar range for which the affinity was higher than that of MK6240 itself. This is, to our knowledge, the first time that a compound other than the tritiated compound has competed with better affinity.

To better understand the binding properties of the different tracers at their binding sites, we analysed the fraction of binding sites targeted by these tracers while competing with the labelled compound. Although both AV-1451 and THK5117 competed with ${ }^{3} \mathrm{H}-\mathrm{MK} 6240$, AV-1451 had the strongest affinity for the ${ }^{3} \mathrm{H}-\mathrm{MK} 6240$ site. It appears that THK compounds have a high affinity for only a low percentage $(37 \%$ for THK5117) of ${ }^{3} \mathrm{H}-\mathrm{MK} 6240$ binding sites (compared with
(TC) lobes. AT8 immunostaining on FC and hippocampus (Hip) from a small section of the right hemisphere is also presented in the figure as a pathology reference. Red $=$ highest binding; blue = lowest binding

$74 \%$ for AV-1451). Moreover, Ni et al. found that unlabelled AV-1451 competed with ${ }^{18} \mathrm{~F}-\mathrm{AV}-1451$ for two binding sites in the temporal cortex of AD brains, and the competitor had a high affinity for $88 \%$ of the sites. In the frontal cortex, this proportion was diminished to $61 \%$ (unpublished data). It seems that brain regions severely affected by $A D$ contain a target displaying sites for which AV-1451 has a much higher affinity than other tracers.

We have previously demonstrated that first-generation tau tracers (THK5351, THK5117 and AV-1451) share similar binding sites on tau deposits [9]. We have observed that MK6240 does not compete with ${ }^{3} \mathrm{H}$-THK5351. Hostetler et al. also reported a lack of competition between MK6240 and ${ }^{3} \mathrm{H}-\mathrm{AV}-1451$ [21]. Interestingly, during the competition study with ${ }^{3} \mathrm{H}$-THK5351, the fraction of sites for which AV-1451 had a high affinity was $35 \%$. AV-1451 had a high affinity for fewer ${ }^{3} \mathrm{H}$-THK5351 sites than ${ }^{3} \mathrm{H}-\mathrm{MK} 6240$ sites, which suggests that the binding properties of MK6240 are closer to those of AV-1451 than to those of THK5351. A summary of the binding properties of the tracers is presented in Fig. 5. We observed that unlabelled MK6240 and AV-1451 had a super-high affinity for a high percentage of the ${ }^{3} \mathrm{H}-\mathrm{MK} 6240$ binding sites and that unlabelled THK5117 had a low affinity for a high percentage of the ${ }^{3} \mathrm{H}-\mathrm{MK} 6240$ binding sites. Similarly, unlabelled AV-1451 had a low affinity for a high percentage of the ${ }^{3} \mathrm{H}$-THK5351 binding sites. Taken together, these competitive binding studies suggest that MK6240 is more similar to AV-1451 than to the THK compound family. 
Fig. 5 Representative schema of the percentages of the ${ }^{3} \mathrm{H}$ MK6240 and ${ }^{3} \mathrm{H}-\mathrm{THK} 5351$ binding sites for which the unlabelled competitors (MK6240, AV1451, THK5351, THK5117) had a super-high or high affinity in competitive binding studies

\section{${ }^{3} \mathrm{H}-\mathrm{MK} 6240$ competitions}

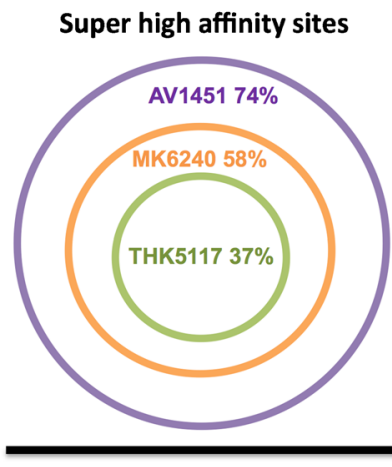

High affinity sites
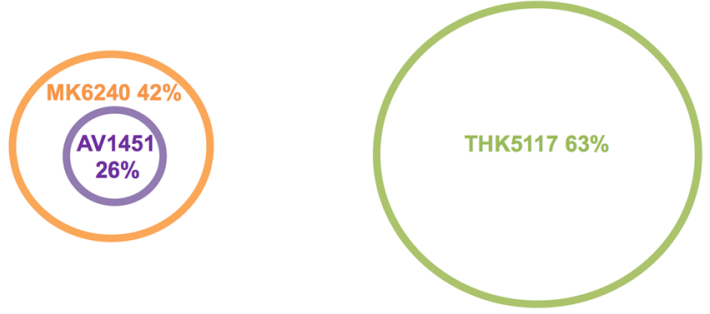

\section{${ }^{3} \mathrm{H}-\mathrm{THK} 5351$ competitions}

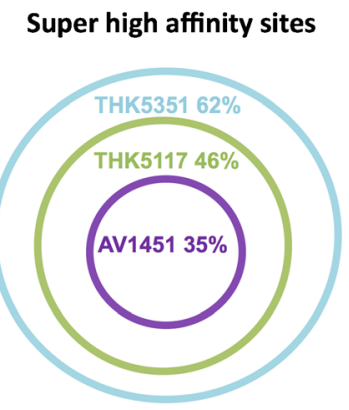

High affinity sites
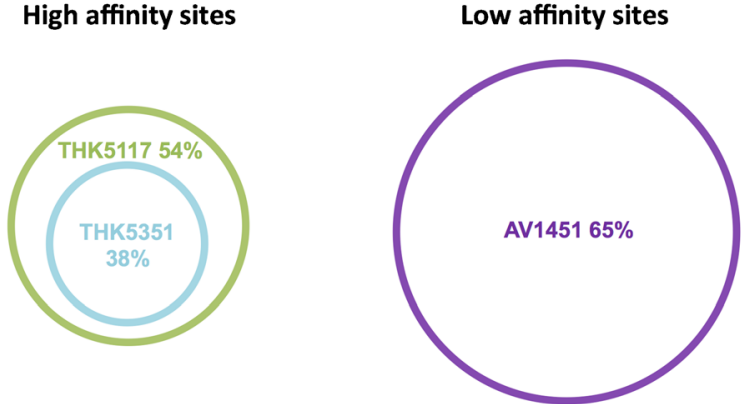

(prefrontal, premotor and inferior parietal cortices) was higher in younger patients than in older patients. A recent study using ${ }^{18} \mathrm{~F}$-MK6240 also showed that 18 F-MK6240 was able to discriminate between early and late stages of the disease in vivo [26]. It is not yet known whether this is the result of differences in the availability of a certain type of tau or maybe a binding site structural change.

Autoradiography ${ }^{3} \mathrm{H}-\mathrm{MK} 6240$ binding studies clearly showed a laminar binding pattern in both frontal and temporal lobes. Specific binding was elevated in the temporal cortex of $\mathrm{AD}$ case $\mathrm{B}$. Binding in the hippocampus was lower in $\mathrm{AD}$ patient $A$ than in $A D$ patient $B$, which can be attributed to atrophy of the anterior part of the hippocampus, as diagnosed by magnetic resonance imaging. The difference in binding between the two samples was confirmed by AT8 pathological immunostaining. When we compared these data with previous data obtained using ${ }^{3} \mathrm{H}$-THK5117 autoradiography on tissue from the same patients, we observed that binding in the frontal cortex of patient 3 was higher than that in patient 2 (see published data [8]). This appears to suggest a difference in the binding properties of THK5117 and MK6240. There was no specific binding in tissue from the control case, who was a 76-year-old female with Braak stage I.

In conclusion, we observed that ${ }^{3} \mathrm{H}-\mathrm{MK} 6240$ had good binding properties in $\mathrm{AD}$ tissue, with high specific binding. First-generation tau PET tracers had at least two binding sites 
in common with MK6240, with binding affinities ranging from super-high to low. The binding affinity visible in PET is in the range of $10 \mathrm{nM}$; in this range, the binding of MK6240 and AV-1451 was similar but with a higher proportion of binding sites for MK6240 than for AV1451. In regional distribution studies using brain homogenates, we saw a clear difference between $\mathrm{AD}$ and control tissue. Almost no specific binding was seen with autoradiography in control tissue. The apparent ability of ${ }^{3} \mathrm{H}-\mathrm{MK} 6240$ to differentiate between EOAD and LOAD tissue may indicate the presence of different tau types in different stages of the disease. A larger sample size is needed to confirm this and to investigate whether it is a load of tau or the kind of tau that differs. This study highlights the complexity of the binding properties of tau tracers from the first and second developmental generations and confirms the need for deeper characterization in order to more fully understand the binding properties of tau PET tracers in AD tissue.

Authors' contributions All authors contributed to the study design. Mona-Lisa Malarte carried out the experiments. The study results were analysed by all authors, all authors helped to draft and revise the manuscript, and all authors approved the final version of the manuscript.

Funding Open access funding provided by Karolinska Institute. This study was funded by the Swedish Foundation for Strategic Research (RB13-0192), the Swedish Research Council (project 05817, 20170295, 2017-06086), the Stockholm County Council-Karolinska Institutet regional agreement on medical training and clinical research (ALF grant), the Swedish Brain Foundation, the Alzheimer Foundation in Sweden, the Foundation for Old Servants, Gun and Bertil Stohne's Foundation, Gunvor och Josef Anérs stiftelsen, the Loo and Hans Osterman Foundation, the Tore Nilsson Foundation, the KI Foundation for Geriatric Diseases and Demensfonden.

Availability of data and material The raw data used in this study are available from the corresponding authors upon reasonable request.

\section{Compliance with ethical standards}

Conflict of interest The authors declare that they have no conflicts of interest.

Ethics approval The study was conducted according to the principles of the Declaration of Helsinki and subsequent revisions. All experiments on autopsied human brain tissue were carried out in accordance with ethical permission obtained from the regional human ethics committee in Stockholm (permission number 2011/962/31-1), and the medical ethics committee of the VU Medical Center for the Netherlands Brain Bank tissue (permission number 1998-06/5).

Consent to participate Previous consent to carry out experiments was given at the time of brain donation, and no supplementary consent was needed for this study.

Consent to publish Previous consent to publish the results of experiments was given at the time of brain donation, and no supplementary consent was needed for this study.
Code availability Not applicable.

Open Access This article is licensed under a Creative Commons Attribution 4.0 International License, which permits use, sharing, adaptation, distribution and reproduction in any medium or format, as long as you give appropriate credit to the original author(s) and the source, provide a link to the Creative Commons licence, and indicate if changes were made. The images or other third party material in this article are included in the article's Creative Commons licence, unless indicated otherwise in a credit line to the material. If material is not included in the article's Creative Commons licence and your intended use is not permitted by statutory regulation or exceeds the permitted use, you will need to obtain permission directly from the copyright holder. To view a copy of this licence, visit http://creativecommons.org/licenses/by/4.0/.

\section{References}

1. Nordberg A. Molecular imaging in Alzheimer's disease: new perspectives on biomarkers for early diagnosis and drug development. Alzheimers Res Ther. 2011;3(6):34.

2. Golde TE, et al. Thinking laterally about neurodegenerative proteinopathies. J Clin Invest. 2013;123(5):1847-55.

3. Villemagne VL, et al. Tau imaging: early progress and future directions. Lancet Neurol. 2015;14(1):114-24.

4. Verwilst $\mathrm{P}$, et al. Shedding light on tau protein aggregation: the progress in developing highly selective fluorophores. Chem Soc Rev. 2018;47(7):2249-65.

5. Okamura N, et al. The development and validation of tau PET tracers: current status and future directions. Clin Transl Imaging. 2018;6(4):305-16.

6. Leuzy A, et al. Tau PET imaging in neurodegenerative tauopathiesstill a challenge. Mol Psychiatry. 2019;24:1112-34

7. Saint-Aubert L, et al. Tau PET imaging: present and future directions. Mol Neurodegener. 2017;12(1):19.

8. Lemoine L, et al. Visualization of regional tau deposits using (3)HTHK5117 in Alzheimer brain tissue. Acta Neuropathol Commun. 2015;3:40.

9. Lemoine L, et al. Comparative binding properties of the tau PET tracers THK5117, THK5351, PBB3, and T807 in postmortem Alzheimer brains. Alzheimers Res Ther. 2017;9(1):96.

10. Chiotis K, et al. Dual tracer tau PET imaging reveals different molecular targets for (11)C-THK5351 and (11)C-PBB3 in the Alzheimer brain. Eur J Nucl Med Mol Imaging. 2018;45(9): 1605-17.

11. Fitzpatrick AWP, et al. Cryo-EM structures of tau filaments from Alzheimer's disease. Nature. 2017;547(7662):185-90.

12. Lemoine $\mathrm{L}$, et al. Cortical laminar tau deposits and activated astrocytes in Alzheimer's disease visualised by (3)H-THK5117 and (3) H-deprenyl autoradiography. Sci Rep. 2017;7:45496.

13. Vermeiren $\mathrm{C}$, et al. The tau positron-emission tomography tracer AV-1451 binds with similar affinities to tau fibrils and monoamine oxidases. Mov Disord. 2018;33(2):273-81.

14. Brosch JR, et al. Tau imaging in Alzheimer's disease diagnosis and clinical trials. Neurotherapeutics. 2017;14(1):62-8.

15. Perez-Soriano A, et al. PBB3 imaging in Parkinsonian disorders: evidence for binding to tau and other proteins. Mov Disord. 2017;32(7):1016-24.

16. Lois $\mathrm{C}$, et al. PET imaging of tau protein targets: a methodology perspective. Brain Imaging Behav. 2019;13(2):333-44.

17. Kuwabara H, et al. Evaluation of (18)F-RO-948 PET for quantitative assessment of tau accumulation in the human brain. J Nucl Med. 2018;59(12):1877-84. 
18. Kroth H, et al. Discovery and preclinical characterization of [18F] PI-2620, a next-generation tau PET tracer for the assessment of tau pathology in Alzheimer's disease and other tauopathies. Eur J Nucl Med Mol Imaging. 2019;46(10):2178-89.

19. Declercq L, et al. Preclinical evaluation of (18)F-JNJ64349311, a novel PET tracer for tau imaging. J Nucl Med. 2017;58(6):975-81.

20. Collier TL, et al. cGMP production of the radiopharmaceutical [(18) F]MK-6240 for PET imaging of human neurofibrillary tangles. J Labelled Comp Radiopharm. 2017;60(5):263-9.

21. Hostetler ED, et al. Preclinical characterization of $18 \mathrm{~F}-\mathrm{MK}-6240$, a promising PET tracer for in vivo quantification of human neurofibrillary tangles. J Nucl Med. 2016;57(10):1599-606.

22. Walji AM, et al. Discovery of 6-(Fluoro-(18)F)-3-(1H-pyrrolo[2,3c]pyridin-1-yl)isoquinolin-5-amine ([(18)F]-MK-6240): a positron emission tomography (PET) imaging agent for quantification of neurofibrillary tangles (NFTs). J Med Chem. 2016;59(10):477889.

23. Murugan NA, et al. Cross-interaction of tau PET tracers with monoamine oxidase B: evidence from in silico modelling and in vivo imaging. Eur J Nucl Med Mol Imaging. 2019;46(6):1369-82.
24. Aguero C, et al. Autoradiography validation of novel tau PET tracer [F-18]-MK-6240 on human postmortem brain tissue. Acta Neuropathol Commun. 2019;7(1):37.

25. Lohith TG, et al. Brain imaging of Alzheimer dementia patients and elderly controls with (18)F-MK-6240, a PET tracer targeting neurofibrillary tangles. J Nucl Med. 2019;60(1):107-14.

26. Pascoal TA, et al. 18F-MK-6240 PET for early and late detection of neurofibrillary tangles. Brain. 2020;143(9): 2818-30.

27. Murugan NA, Nordberg A, Agren H. Different positron emission tomography tau tracers bind to multiple binding sites on the tau fibril: insight from computational modeling. ACS Chem Neurosci. 2018;9(7):1757-67.

28. Scholl M, et al. Distinct 18F-AV-1451 tau PET retention patterns in early- and late-onset Alzheimer's disease. Brain. 2017;140(9): 2286-94.

Publisher's note Springer Nature remains neutral with regard to jurisdictional claims in published maps and institutional affiliations. 